\title{
The use of intravenous immunoglobulin therapy in a tertiary pediatric hospital
}

\author{
Ali Güngör, MD, Neşe Yaral, MD.
}

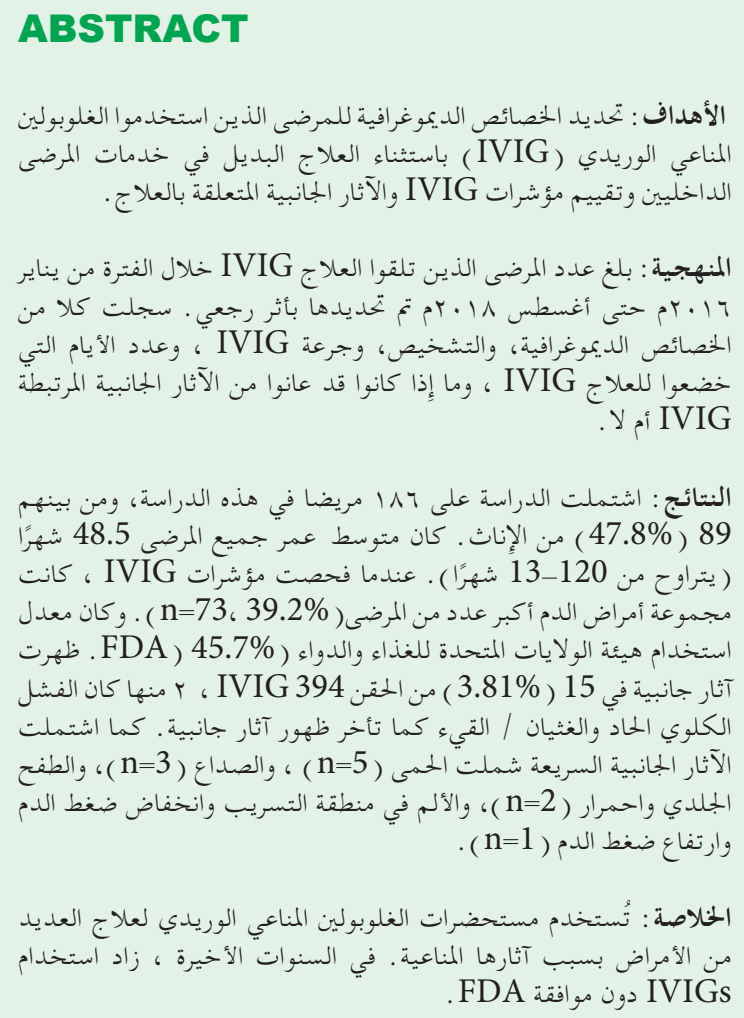

Objectives: To determine demographic features of patients provided intravenous immunoglobulin (IVIG) excluding replacement therapy in the inpatient services and to evaluate indications for IVIG, and side effects related to therapy.

Methods: Patients who received IVIG therapy between January 2016 and August 2018 were retrospectively identified. The demographic features, diagnosis, IVIG dose, number of days they underwent IVIG therapy, and whether or not they experienced IVIG-related side effects were recorded.

Results: A total of 186 patients were included in this study, and of these, $89(47.8 \%)$ were females. The median age of all of the patients was 48.5 months old (range 13-120 months). When the IVIG indications were examined, the hematological disease group had the highest number of patients $(\mathrm{n}=73,39.2 \%)$. The US Food and Drug Administration (FDA) labeled use rate was $45.7 \%$. Side effects were seen in 15 (3.81\%) of the 394 IVIG infusions, 2 of which were acute kidney failure and nausea/vomiting as delayed onset side effects. The rapid onset side effects included fever $(n=5)$, headache $(n=3)$, rash and redness $(n=2)$, and pain in the infusion area, hypotension, and hypertension $(\mathrm{n}=1)$.

Conclusion: Intravenous immunoglobulin preparations are used for the treatment of many diseases due to their immunoregulatory effects. In recent years, the use of IVIGs without FDA approval has been increasing.

Keywords: adverse effects, child, intravenous immunoglobulin, immunoregulatory

Saudi Med J 2020; Vol. 41 (2): 163-167 doi: 10.15537/smj.2020.2.24877

From the Department of Pediatrics (Gungor) and form the Department of Pediatric Hematology and Oncology (Yarali), Ankara Child Health and Diseases Hematology Oncology Training and Research Hospital, Anakara, Turkey.

Received 4th November 2019. Accepted 25th December 2019.

Address correspondence and reprint request to: Dr. Ali Güngör, Department of Pediatrics, University of Health Sciences, Ankara Child Health and Diseases Hematology Oncology Training and Research Hospital, Ankara, Turkey. E-mail: gungorali19@gmail.com

ORCID ID: https://orcid.org/0000-0003-4139-3480

Tntravenous immunoglobulin (IVIG) preparations 1 are plasma products obtained from healthy donors. Intravenous immunoglobulin therapy is primarily used as a replacement therapy for immunodeficient patients, but it can also be used for the treatment of many diseases, at high doses, due to its anti-inflammatory and immunoregulatory effects. ${ }^{1,2}$ The indications for US 
Food and Drug Administration (FDA) approved IVIG use include several diseases, such as primary humoral immunodeficiency, immune thrombocytopenic purpura (ITP), Kawasaki disease (KD), chronic lymphocytic leukemia, and multifocal motor neuropathy. It has also been proven that IVIG therapy is beneficial for the treatment of many other diseases, such as Guillain-Barre syndrome (GBS), neonatal sepsis, neonatal blood type incompatibility, autoimmune hemolytic anemia (AHIA), and Stevens-Johnson syndrome. ${ }^{3-5}$ Although IVIGs are used at dosages of $400-500 \mathrm{mg} / \mathrm{kg}$ in replacement therapy, higher doses are used for immunoregulatory treatments. ${ }^{1,6}$

Several early onset side effects of IVIG use have been observed, including fever, rash, headache, nausea, vomiting, and myalgia, as well as late onset and severe side effects, including aseptic meningitis, acute kidney failure, thromboembolic events, hemolytic anemia, and myocardial infarctions. Although the incidence of IVIG-related side effects differs among previous studies, it has been reported to range from 3-20\%.7-10

The aim of this study was to determine the demographic features of inpatients receiving IVIG therapy for immunoregulatory treatment, and to evaluate the indications for and side effects of IVIG therapy in a tertiary pediatrics hospital.

Methods. Patients who received IVIG therapy between January 2016 and August 2018 at the University of Health Sciences, Ankara Child Health and Diseases Hematology Oncology Training and Research Hospital (which is a tertiary pediatrics hospital), Ankara, Turkey, were retrospectively identified from the patient file registry system. Patients who underwent IVIG treatments as replacement therapy were excluded from the study. Additionally, 17 patients were excluded due to insufficient information. Therefore, a total of 186 patients with complete data available from the file registry system were included in this study. The following information was recorded for each patient: demographic features, diagnosis, inpatient service in which they were followed-up, IVIG dosage, number of IVIG treatment days, and whether or not IVIG-related side effects occurred. It was determined that all of the patients who underwent IVIG therapy were provided antipyretics and antihistamines in order

Disclosure. Authors have no conflict of interests, and the work was not supported or funded by any drug company. to reduce the side effects. In order to determine the IVIG-related side effects, we searched for the number of hospitalization days after the IVIG therapy. Those patients with follow-ups of longer than 2 weeks after the last IVIG dose were gathered into a single group. The side effects that occurred within the first 6 hours after the IVIG infusion were considered to be early onset side effects, and those that occurred between 6 hours and one week afterward were considered to be delayed onset side effects. The patients' diagnoses were divided into 7 groups: neurological, hematological, dermatological, cardiological, rheumatic, infectious, and neonatal diseases. The use of IVIGs for the treatment of primary humoral immunodeficiencies, ITP, KD, chronic lymphocytic leukemia, and multifocal motor neuropathy was found to be approved by the FDA.

The protocol for this study was approved by the University of Health Sciences, Ankara Child Health and Diseases Hematology Oncology Training and Research Hospital Ethics Committee (number 2018/168).

Statistical analysis. The statistical data was calculated using the Statistical Package for Social Sciences (SPSS) version 18.0 for Windows (SPSS Inc, Chicago, IL, USA). A descriptive statistical analysis was conducted, and because the age at diagnosis was not normally distributed, the data was expressed as the median and interquartile range. The qualitative data was expressed as number and percentage. The Chi-squared test was used to compare the qualitative data, and the Pearson correlation test was used for the correlation analysis. A $p<0.05$ was considered significant.

Results. A total of 186 patients were included in this study and they received a total of 394 IVIG doses. Eighty-nine $(47.8 \%)$ of the patients were females and 97 (52.2\%) were males, with a median age of 48.5 months old (range 13-120 months). Ninety-six (51.6\%) patients received a single IVIG dose, and the remaining patients received between $2-5$ doses. With regard to their nationalities, $165(88.7 \%)$ patients were Turkish, $16(8.6 \%)$ were Syrian, $4(2.2 \%)$ were Iraqi, and one (0.5\%) was Afghan.

Most of the patients were hospitalized in the hematology-oncology service $(32.8 \%)$, followed by the older children's service $(17.2 \%)$, and the pediatric intensive care unit $(15.1 \%)$ (Table 1$)$.

When the indications for the IVIG administration were examined, $39.2 \%$ patients were in the hematological disease group, $16.7 \%$ were in the cardiological disease group, $13.5 \%$ were in the rheumatic disease group, and $11.8 \%$ were in the neurological disease group (Table 2). The FDA labeled usage rate was $45.7 \%(n=85)$. 
Table 1 - Distribution of services where patients received intravenous immunoglobulin therapy.

\begin{tabular}{|c|c|}
\hline Service & n (\%) \\
\hline Hematology-oncology service & $61(32.8)$ \\
\hline Older children service ( $2-18$ years) & $32(17.2)$ \\
\hline Pediatric intensive care unit & $28(15.1)$ \\
\hline Neonatal intensive care unit & $18 \quad(9.7)$ \\
\hline Infectious diseases service & $18 \quad(9.7)$ \\
\hline Burn + pediatric surgery intensive care unit & $15 \quad(8)$ \\
\hline Infant service (1-24 months) & $14(7.5)$ \\
\hline Total & $186(100)$ \\
\hline
\end{tabular}

The 186 patients included in this study received a total of 394 IVIG doses. Of these treatments, 211 $(53.6 \%)$ doses of $0.4-0.5 \mathrm{~g} / \mathrm{kg}$ were provided, 82 $(20.8 \%)$ doses of $0.8 \mathrm{~g} / \mathrm{kg}$ were provided, 74 (18.8\%) doses of $1 \mathrm{~g} / \mathrm{kg}$ were provided, and 27 (6.9\%) doses of $2 \mathrm{~g} / \mathrm{kg}$ were provided. Side effects were observed in $15(3.8 \%)$ of a total of 394 IVIG doses, 2 of which were provided as $1 \mathrm{~g} / \mathrm{kg}, 2$ as $2 \mathrm{~g} / \mathrm{kg}, 5$ as $0.4-0.5 \mathrm{~g} /$ $\mathrm{kg}$ and 6 as $0.8 \mathrm{~g} / \mathrm{kg}$. When dose-dependent side effects were examined; side effects were seen in 2 of $27(7.4 \%)$ patients who received IVIG as $2 \mathrm{~g} / \mathrm{kg}$, in 2 of $74(2.7 \%)$ patients who received $1 \mathrm{~g} / \mathrm{kg}$, in 6 of 82 (7.31\%) patients who received $0.8 \mathrm{~g} / \mathrm{kg}$, and in 5 of $211(2.4 \%)$ patients who received $0.4 \mathrm{~g} / \mathrm{kg}$. There were no significant differences between IVIG dose and side effects $(p=0.161)$.

Two of the side effects were acute kidney failure and nausea/vomiting as delayed onset side effects. The rapid onset side effects included fever $(n=5)$, headache $(n=3)$, rash and redness $(n=2)$, and pain in the infusion area, hypotension, and hypertension $(\mathrm{n}=1)$. Of the 15 patients in whom side effects were observed, 7 (46.7\%) of the patients were provided IVIG treatments for FDA approved diseases and $8(53.33 \%)$ were provided IVIG treatments without FDA approval. Moreover, $32(17.2 \%)$ of the patients were discharged one day after receiving the IVIG therapy, 98 (52.69\%) were discharged between 2-7 days after the IVIG therapy, and $30(16.1 \%)$ were discharged between 8-14 days after the therapy. Twenty-six (14\%) patients were hospitalized and followed-up for more than 14 days following the IVIG therapy.

Discussion. In addition to replacement therapy, IVIG therapy is commonly used to treat several diseases for which it has not been approved by the FDA due to its immunoregulatory effects. In a Malaysian study in which 115 pediatric patients were evaluated, the FDA labeled usage rate was 53\%; however, this rate was $70 \%$ in one study from Turkey conducted in a pediatric
Table 2 - Indications for intravenous immunoglobulin therapy.

\begin{tabular}{lrr}
\hline Disease group & $\mathbf{n}(\%)$ \\
\hline Hematologic diseases & $73(39.2)$ \\
ITP ( acute) & 47 & $(25.2)$ \\
ITP (chronic) & 15 & $(8.1)$ \\
Autoimmune hemolytic anemia & 6 & $(3.2)$ \\
Hemophagocytic lymphohistiocytosis & 5 & $(2.7)$ \\
Cardiological diseases & & \\
Myocarditis & 31 & $(16.7)$ \\
Rheumatic diseases & 25 & $(13.5)$ \\
Kawasaki disease & 23 & $(12.4)$ \\
Dermatomyositis & 2 & $(1.1)$ \\
Neurologic diseases & 22 & $(11.8)$ \\
GBS & 12 & $(6.4)$ \\
Autoimmune limbic encephalitis & 7 & $(3.8)$ \\
Multiple sclerosis & 2 & $(1.1)$ \\
Resistant epilepsy & 1 & $(0.5)$ \\
Infectious diseases & & \\
Sepsis & 16 & $(8.6)$ \\
Newborn disease & 14 & $(7.5)$ \\
Neonatal sepsis & 3 & $(1.6)$ \\
Alloimmune thrombocytopenia & 3 & $(1.6)$ \\
Hemolytic anemia (ABO-RH incompatibility) & 8 & $(4.3)$ \\
Dermatologic diseases & 5 & $(2.7)$ \\
Steven-Johnson syndrome & 4 & $(2.2)$ \\
DRESS & 1 & $(0.5)$ \\
\hline ITP: immune thrombocytopenic purpura, GBS: Guillain-Barre \\
syndrome, DRESS: drug rash with eosinophilia and systemic \\
symptoms, ABO-RH: blood groups & & \\
\hline
\end{tabular}

infectious diseases clinic. ${ }^{11,12}$ In our study, the FDA labeled usage rate was $45.7 \%$. This lower FDA labeled usage rate when compared to other studies may have been due to the fact that immunodeficiency conditions, which are the primary IVIG usage area, were not included in this study.

Hematological diseases are among those diseases for which IVIGs are commonly used, including immune thrombocytopenic purpura, AIHA, hemophagocytic lymphohistiocytosis (HLH), and bone marrow transplantation cases. ${ }^{13,14}$ The most common diseases for which IVIGs were used were hematological diseases. Intravenous immunoglobulins were provided to 62 patients with diagnoses of ITP, 6 patients with AIHA, and 5 patients with HLH. This may be attributed to the fact that the number of patients followed up was high, because the hematology oncology unit of our hospital is a large center with a 50-bed capacity.

Cardiac complications decrease if IVIGs are provided within the first 10 days of $\mathrm{KD}$, and it is recommended that this therapy be used at higher doses $(2 \mathrm{~g} / \mathrm{kg} /$ day $)$ when compared to replacement therapy. ${ }^{15,16}$ In our study, $23(12.4 \%)$ patients received IVIG therapy due to $\mathrm{KD}$, and all of the patients were provided doses of $2 \mathrm{~g} / \mathrm{kg}$. 
Intravenous immunoglobulin use is common in neurological disease cases. Previous studies have shown that IVIG therapy is as effective as plasmapheresis therapy, especially in GBS. In addition, IVIGs may be helpful in other neurological diseases, such as multiple sclerosis, autoimmune encephalitis, and resistant epilepsy. ${ }^{11,17}$ In our study, 22 (11.8\%) patients received IVIG treatments for neurological diseases, and the most common indication in this groups was GBS $(n=12)$.

One of the diseases for which IVIG preparations are used without FDA approval is sepsis, especially during the neonatal period. In one study from Egypt that included sepsis patients in a pediatric intensive care unit, IVIG therapy was provided as an adjuvant therapy for sepsis treatment, and it was found to have positive effects on the length of stay in the intensive care unit. ${ }^{18}$ In our study, 19 (10.2\%) patients received IVIG therapy to support sepsis treatment. Although 7 of these patients died, the remaining 12 patients recovered from sepsis. Among the patients that died, 4 had severe burns on the body, 2 had neonatal sepsis, and one was a 4-month-old patient who underwent surgery for jejunal atresia and exhibited severe malnutrition and ileus. In our study, we only evaluated the patients who were provided IVIG therapy; therefore, the utility of the IVIG therapy was not evaluated with regard to sepsis treatment.

The use of IVIGs in the treatment of acute myocarditis in pediatric patients has been shown to have positive effects on left ventricular functions and survival, and high dosages $(2 \mathrm{~g} / \mathrm{kg})$ have been recommended. ${ }^{19,20}$ In our study, $31(16.7 \%)$ patients with diagnoses of myocarditis received IVIG therapy at dosages of $2 \mathrm{~g} / \mathrm{kg}$.

The rate of IVIG-related side effects varies among studies. In one study from Turkey, the rate was $9.3 \%$, but in a study from Malaysia, it was $6.1 \% .^{11,12}$ In another study from the Philippines that evaluated 77 patients, IVIG-related side effects were found in 32\% of the cases, with the most common side effects being fever and rash. ${ }^{21}$ In our study, side effects were seen in $15(3.8 \%)$ of the 394 IVIG infusions. Two of the patients exhibited acute kidney failure and nausea/ vomiting as delayed onset side effects. The rapid onset side effects included: fever in 5 patients; headache in 3 patients; rash and redness in 2 patients, and pain in the infusion area hypotension; and hypertension in one patient. Aseptic meningitis and anaphylaxis are among the severe side effects of IVIG therapy, but these were not found in any of our patients. The lower rate of side effects in our study when compared to the literature may be attributed to the following: all of the patients received premedication before therapy, the infusions were usually provided slowly over 8 hours, and some of the patients were discharged a short time after receiving the IVIG therapy, and they could not be evaluated for delayed onset side effects.

Study limitations. This study was designed as a retrospective study with data from a single center. Moreover, an efficacy comparison could not be performed with patients who did not receive IVIG therapy.

In conclusion, IVIG preparations are used for the treatment of many diseases due to their immunoregulatory effects. In recent years, the use of IVIG therapy without FDA approval has been increasing. Decisions on this treatment should be made while considering its costs and side effects. Further studies are needed to evaluate the efficacy of this treatment in patients without definitive indications.

Acknowledgment. The authors gratefully acknowledge Scribendi (www.scribendi.com) for English language editing.

\section{References}

1. Prasad AN, Chaudhary S. Intravenous immunoglobulin in pediatrics: a review. Med J Armed Forces India 2014; 70: 277-280.

2. Zülfikar B, Koç B. Use of intravenous immunoglobulin in pediatric practice. Turk Pediatri Ars 2014; 49: 282-288.

3. Galal NM. Pattern of intravenous immunoglobulins (IVIG) use in a pediatric intensive care facility in a resource limited setting. Afr Health Sci 2013; 13: 261-265.

4. Garcia-Lloret M, McGhee S, Chatila TA. Immunoglobulin replacement therapy in children. Immunol Allergy Clin North Am 2008; 28: 833-849.

5. Perez EE, Orange JS, Bonilla F, Chinen J, Chinn IK, Dorsey $\mathrm{M}$, et al. Update on the use of immunoglobulin in human disease: A review of evidence. J Allergy Clin Immunol 2017; 139: S1-S46.

6. Wong PH, White KM. Impact of immunoglobulin therapy in pediatric disease: a review of immune mechanisms. Clin Rev Allergy Immunol 2016; 51: 303-314.

7. Guo Y, Tian X, Wang X, Xiao Z. Adverse effects of immunoglobulin therapy. Front Immunol 2018; 9: 1299.

8. Pierce LR, Jain N. Risks associated with the use of intravenous immunoglobulin. Transfus Med Rev 2003; 17: 241-251.

9. Cherin P, Marie I, Michallet M, Pelus E, Dantal J, Crave JC, et al. Management of adverse events in the treatment of patients with immunoglobulin therapy: a review of evidence. Autoimmun Rev 2016; 15: 71-81.

10. Akman AO, Kara FK, Koksal T, Cakir BC, Karagol C, Saylı T. Association of hemolysis with high dose intravenous immunoglobulin therapy in pediatric patients: an open-label prospective trial. Transfus Apher Sci 2017; 56: 531-534.

11. Gayretli Aydın ZG, Tanır G. Clinical indications of intravenous immunoglobulin use in Pediatric Infectious Diseases Clinic. $J$ Pediatr Inf 2017; 11: e148-e153.

12. Toh CC, Islahudin F, Adli A, Noraida Mohamed S. Intravenous immunoglobulin therapy among pediatric patients: labeled and off-labeled indications. Braz J Pharm Sci 2018; 54: e0008. 
13. Jolles S, Sewell WA, Misbah SA. Clinical uses of intravenous immunoglobulin. Clin Exp Immunol 2005; 142: 1-11.

14. Anderson D, Ali K, Blanchette V, Brouwers M, Couban S, Radmoor P, et al. Guidelines on the use of intravenous immune globulin for hematologic conditions. Transfus Med Rev 2007; 21: S9-S56.

15. Hedrich CM, Schnabel A, Hospach T. Kawasaki Disease. Front Pediatr 2018; 6: 198.

16. Oates-Whitehead RM, Baumer JH, Haines L, Love S, Maconochie IK, Gupta A, et al. Intravenous immunoglobulin for the treatment of Kawasaki disease in children. Cochrane Database Syst Rev 2003; CD004000.

17. Gadian J, Kirk E, Holliday K, Lim M, Absoud M. Systematic review of immunoglobulin use in paediatric neurological and neurodevelopmental disorders. Dev Med Child Neurol 2017; 59: 136-144.
18. El-Nawawy A, El-Kinany H, Hamdy El-Sayed M, Boshra N. Intravenous polyclonal immunoglobulin administration to sepsis syndrome patients: a prospective study in a pediatric intensive care unit. J Trop Pediatr 2005; 51: 271-278.

19. Prasad AN, Chaudhary S. Intravenous immunoglobulin in children with acute myocarditis and/or early dilated cardiomyopathy. Indian Pediatr 2014; 51: 583-584.

20. Farinha IT, Miranda JO. Myocarditis in paediatric patients: unveiling the progression to dilated cardiomyopathy and heart failure. J Cardiovasc Dev Dis 2016; 3: E31.

21. Palabrica FR, Kwong SL, Padua FR. Adverse events of intravenous immunoglobulin infusions: a ten-year retrospective study. Asia Pac Allergy 2013; 3: 249-256.

\section{References}

* References should be primary source and numbered in the order in which they appear in the text. At the end of the article the full list of references should follow the Vancouver style.

* Unpublished data and personal communications should be cited only in the text, not as a formal reference.

* The author is responsible for the accuracy and completeness of references and for their correct textual citation.

* When a citation is referred to in the text by name, the accompanying reference must be from the original source.

* Upon acceptance of a paper all authors must be able to provide the full paper for each reference cited upon request at any time up to publication.

* Only 1-2 up to date references should be used for each particular point in the text.

Sample references are available from:

http://www.nlm.nih.gov/bsd/uniform_requirements.html 